\title{
Issues of Mass and Count: Dealing with 'Dual-Life' Nouns
}

\author{
Tibor Kiss \\ Ruhr-Universität Bochum \\ 44801 Bochum, Germany \\ tiborelinguistics.rub.de
}

\author{
Francis Jeffry Pelletier \\ University of Alberta \\ Edmonton, Canada \\ francisp@ualberta.ca
}

\author{
Halima Husić \\ Ruhr-Universität Bochum \\ 44801 Bochum, Germany \\ husic@linguistics.rub.de
}

\author{
Johanna Poppek \\ Ruhr-Universität Bochum \\ 44801 Bochum, Germany \\ poppek@linguistics.rub.de
}

\begin{abstract}
The topics of + MASS and +COunT have been studied for many decades in philosophy (e.g., (Quine, 1960; Pelletier, 1975)), linguistics (e.g., (McCawley, 1975; Allan, 1980; Krifka, 1991)) and psychology (e.g., (Middleton et al., 2004; Barner et al., 2009). More recently, interest from within computational linguistics has studied the issues involved (e.g., (Pustejovsky, 1991; Bond, 2005; Schmidtke and Kuperman, 2016)), to name just a few. As is pointed out in these works, there are many difficult conceptual issues involved in the study of this contrast. In this article we study one of these issues - the "Dual-Life" of being simultaneously +MASs and +COUNT - by means of an unusual combination of human annotation, online lexical resources, and online corpora.
\end{abstract}

\section{Background}

The standard story of +MAss and + COUNT usually starts with some examples of nouns of both sorts.

- +Count: car, dog, idea, university, belief, ...

- +MAss: water, garbage, advice, oil, admiration, knowledge...

These examples are usually accompanied by some syntactic tests for + MASS and +COUNT:

- Count terms can be pluralized, occur with indefinite determiner, allow numeral modifiers, occur with the quantifiers each, every, .... Mass terms can't do any of these.

- Always occurring in the singular, mass terms occur with "measure" terms (e.g., much), with the quantifiers most, all and the unstressed some, and bare in (e.g.) subject position with singular verb agreement. Singular count terms can't do any of these.
These lead to such comparisons as

(1) a. a car, four ideas, each university

b. *a water, *four garbage, *each admiration

c. all garbage, most water, advice is helpful

d. *all dog, *most university, *ar is fast

It can be seen from this brief (and partial) description of the +MASS/+COUNT distinction that the presumption is that the distinction applies to lexical nouns and that it is exhaustive and exclusive every such noun is either + COUNT or +MASs, and no noun is both.

Our account will deny all these presumptions, and in doing so will open what we find to be a much more plausible account of various mysterious phenomena surrounding +MASS and +COUNT (although in this study we just examine the "duallife" case).

\section{Some Background Methodological Issues}

A fundamental feature of (and, we think, a problem with) with the "usual story" is that it presumes that the locus or home of +MASs and +COUNT is the lexical noun. This is a feature of modern analyses as well as the older ones despite the fact that even in the oldest of the works we find remarks cautioning against this, such as (Quine, 1960)'s ambiguous Mary put a little chicken into the salad and cautionary remarks by others that such sentences as We had crocodile for supper last night! are completely normal. And to the claim that this "changes the sense" of chicken from + Count to +Mass, (Pelletier, 1975, p.456) remarked "Such a claim makes clear that either (1) surface structure is not what the criteria are talking about or (2) we need to distinguish not between mass and count nouns but between mass and count senses of nouns." This sort of remark can be found throughout the literature on +MASs/+COUNT; however, dic- 
tionaries in fact do not usually make this distinction. The only relevant sense of crocodile in WordNet (Miller, 1995; Miller and Fellbaum, 2007) and Webster's New Collegiate Dictionary is "large voracious aquatic reptile having a long snout with massive jaws and sharp teeth and a body covered with bony plates; of sluggish tropical waters". In fact, with only a few exceptions, the relationship between an animal and its flesh used as food (when it is the same noun for each) does not generate new senses in dictionaries (nor WordNet). Somehow, both of the alleged meanings are contained in the same sense.

One might postulate (and many theorists have, e.g., (Bunt, 1985; Payne and Huddleston, 2002)) that there are some background "rules" that can apply to a basic meaning of a noun and which will generate the related sense that describes the opposite value of the +MASS/+COUnT dimension. Suggestions include rules for grinding (armadillo all over the road), portioning (Order me a beer), sorting (eight beers on tap), evaluating (too much car for the average driver) and others. However, it has seemed clear to most theorists that this sort of strategy wouldn't be able to account for all the varied ways that +MASs and +COUNT senses of a given noun might be related. Furthermore, for many socalled abstract nouns, it is not even clear what it is that makes a meaning be +MASs or +COUNT.

Another problem is that researchers looking into the issues involved in +MASs/+COUNT tend to use their own intuitions, based on a very limited number of data points (that is, a very limited group of words and their meanings). The use of largescale resources is relatively rare (although see (Baldwin and Bond, 2003; Grimm, 2014; Katz and Zamparelli, 2012; Kulkarni et al., 2013)). And the use of dictionary resources is also rare, meaning that researchers rely on their own "intuitions into meaning" when it comes to issues of + COUNT and +MAss. Although many researchers have pointed to these sorts of limitations, none have actually investigated the actual senses of nouns as they appear in large resources (e.g., dictionaries), nor have they investigated what it means for a specific sense to be "dual-life" - i.e., to be both + mass and +COUNT.

For these sorts of reasons, we have decided to investigate the possibility that the locus of + MAss and +COunt should be a given sense of a noun. But a consequence of this will be that some senses are both + COUnT and +MASs, as for instance, our examples beer and crocodile. Even if we wish to retain the "semantic conversion/coercion" rules mentioned above and thus excuse these from being both +MASs and +COUNT, there are many others (as we will show below) that do not lend themselves to such coercions. So one of our goals is to display many individual senses of nouns (we call these "noun-senses") that are both +MAss and +COUnT;. The usual name for a theory that allows something to be both +MASS and + COUNT is "duallife" - "dual-life nouns" if the locus is the noun, but "dual-life noun-senses" for our viewpoint.

\section{What We Did}

We used the American National Corpus (ANC: (Ide and Suderman, 2004; Ide, 2008)), parsed with the Stanford NLP Group (http://nlp. stanford.edu) parser (Chen and Manning, 2014). We then intersected the ANC's set of nouns with those in WordNet to form a repository of nouns for which there were definitions (in WordNet). ${ }^{1}$ We employed four graduate linguistics students to (independently) evaluate the extent to which each of the WordNet senses thus chosen could be used in certain contexts (we call these tests the 'syntactic tests') and whether, given the answers to some of the syntactic tests, certain implications follow from their use (we call these the 'semantic tests'). There are six of these tests in all, four syntactic and two semantic, chosen for their relevance to various of the issues that are salient in the studies of + MAss and +COUNT terms. Table 1 gives the bare-bones outline of the annotators' tasks, which ask whether the annotators can construct sentences obeying the syntactic patterns specified, while maintaining the NOUN's meaning to be the one under investigation. Table 2 shows some noun-senses and how they fare with the tests. We discovered that there was very significant inter-annotator agreement in these answers. We have approximately 13,000 annotated and agreed-upon noun-senses. Interannotator agreement, as measured by Krippendorff's $\alpha,=0.755$, which (Artstein and Poesio, 2008, pp. 576, 591) define as highly reliable.

\footnotetext{
${ }^{1}$ We employed the ANC as a natural corpus because we also wished to investigate the actual senses in use. Our annotators characterized the MASS/COUNT feature of the various senses, but we wished then to see which of these senses were actually used in what contexts. That aspect of our research is not reported in the present paper.
} 


\begin{tabular}{|c|c|}
\hline Syn 1: & lar form appear together with more? \\
\hline Sem & $\begin{array}{l}\text { If Syn } 1=\text { yes, is the comparison based on number of entities, or another mode of } \\
\text { measurement? }\end{array}$ \\
\hline Syn 2: & Can the noun-sense pair in its plural form appear together with more? \\
\hline Sem 2: & If Syn $2=$ yes, is the sentence equivalent to a sentence with an explicit classifier? \\
\hline Syn 3: & $\begin{array}{l}\text { Can the noun-sense pair in its singular form and combined with an indefinite determiner } \\
\text { be the syntactic subject of a definition or characterization? }\end{array}$ \\
\hline Syn 4: & $\begin{array}{l}\text { Can the noun-sense pair in its singular form but without a determiner be the syntactic } \\
\text { subject of a definition or characterization? }\end{array}$ \\
\hline
\end{tabular}

Table 1: Four syntactic tests and two semantic tests annotators answered for each noun-sense

\begin{tabular}{|c|c|c|c|c|c|c|c|}
\hline Noun & WordNet description & Syn 1 & Sem 1 & Syn 2 & Sem 2 & Syn 3 & Syn 4 \\
\hline car\#1 & $\begin{array}{l}\text { a motor vehicle with four } \\
\text { wheels }\end{array}$ & no & na & yes & $\neg$ equiv. & yes & no \\
\hline fruitcake\#1 & a whimsically eccentric person & no & na & yes & $\neg$ equiv. & yes & no \\
\hline fruitcake\#2 & $\begin{array}{l}\text { a rich cake containing dried } \\
\text { fruit and nuts }[\ldots]\end{array}$ & yes & $\neg$ num. & yes & $\neg$ equiv. & yes & yes \\
\hline lingerie\#1 & $\begin{array}{l}\text { women's underwear and } \\
\text { nightclothes }\end{array}$ & yes & num. & na & na & no & yes \\
\hline whiskey\#1 & $\begin{array}{l}\text { a liquor made from fermented } \\
\text { mash of grain }\end{array}$ & yes & $\neg$ num. & yes & equiv. & no & yes \\
\hline
\end{tabular}

Table 2: Examples of Test Outcomes.

Further details on the texts, the various senses and the annotation process can be found in (Kiss et al., 2014). Some other aspects of the general research effort are in (Kiss et al., 2016).

Each noun-sense thus gets some unique pattern of answers, which we can represent as an ordered six-tuple of answers (we use the ordering given in Table 1). We extracted and processed the annotators' responses using $\mathbf{R}$ (https : \cran. $r$-project.org), allowing us not only to process the resulting answers given by our annotators, but also to aid in the inner-annotator agreement evaluation. A side effect of using $\mathbf{R}$ numerical names to the groups of senses that have the same six-tuple of answers. In this study we focus on one of these groups ("Classes"); $\mathbf{R}$ gave it the name "726", which we have kept (even though "duallife" might have been a more informative choice of name).

There are three possible answers for each of the six Tests: yes, no, and not applicable (but we sometimes use 'num' and 'eq', together with negations), hence 729 possible classes. But the questions are not independent of one another, and in fact there are only 80 independently possible classes. Our annotators found there to be 18 ac- tual classes to be populated with noun-senses, out of these 80 possible classes.

\section{4 “Dual Life" senses}

Remembering now that we are describing senses of nouns, as identified by WordNet, what sort of noun-senses manifest this duality of being both +MASS and +COUNT? Or put more accurately, since we test the senses by determining answers to questions that have that sense used in a full noun phrase, what sort of noun-senses manifest the possibility of occurring in both +MASS NPs and also in +COUNT NPs?

Our main group of such senses is called Class 726 by $\mathbf{R}$. This group has the profile $<$ yes, $\neg$ num,yes, $\neg$ equiv,yes,yes $>$. That is,

(2) a. This noun-sense can be used in the singular with more. <e.g., John has more X than Mary>

b. This amount of $\mathrm{X}$ is not based on instances of $X$.

c. It can be used in the plural <e.g., John has more Xs than Mary>

d. The Xs are not equivalent to any classifier $+\mathrm{X}<$ e.g., not equivalent to more 
cups (or kinds) of $X>$

e. It can be used with an indefinite singular determiner definition <e.g., An X is a (some definition) $>$

f. It can be used in the singular without a determiner definition <e.g., $X$ is (some definition)>

Class 726 contains 162 different senses of nouns. There are two different broad categories that we can distinguish within this group of duallife noun-senses. The two basic types are:

\section{Senses of Nominally-Oriented nouns \\ 2. Senses of Verbally-Oriented nouns}

There are 57 senses of Type 1 and 96 senses of Type 2. There are thus 8 other senses. 3 of which seem to fit into both categories equally, and 5 that do not seem to be of either Type.

\subsection{Nominally-Oriented Noun Senses}

We start with the Type 1 senses. Unlike the nouns that give rise to the Type 2 senses, the nouns behind these senses are not formed from other parts of speech: they are either simple nouns on their own or else nouns compounded from nouns and possibly other (non-verb) parts of speech.

Some of the Nominally-Oriented Noun senses are in groups that have been discussed in the literature before. One Nominally-Oriented noun type that has long received play in the mass-count literature is that associated with food. ${ }^{2}$ For animal noun-senses other than pig, cow, e.g., alligator, the animal-designating "meaning" is +COUNT while the flesh-designating "meaning" is +MAss. A special version of this occurs when the particular amount or type of the meat is typically cooked or served as a unit, then such a unit gets called by a special +COUNT term and that special term acquires a +COUnT "meaning" for the meat that comprises it: We had a steak for dinner/We had steak for dinner; Mary cooked a ham for Easter/Mary served ham for supper; George bought a large roast/George

\footnotetext{
${ }^{2}$ As remarked above, dictionaries and other lexical sources (in particular, WordNet) take these differing ways to interpret a word like alligator to be parts of one and the same sense. Yet in writing about them, we wish to be able to discuss the differences that are internal to a sense. So, we have decided to use the term "meaning" (with double quote marks) when we wish to discuss the different interpretations that can be given to a single sense (or alternatively put, the different ways that a single sense can be used).
}

had leftover roast for a week. We give one example of a noun-sense that is in this group, and then list the nouns for the other 8 senses of the members of this subgroup.

(3) a. fruitcake\#2: a rich cake containing dried fruit and nuts and citrus peel [...]

b. cake\#3, casserole\#1, ham\#1, marshmallow\#1, melon\#2, pizza\#1, salad\#1, steak\#1

(Rothstein, 2010) brought attention to a class of dual-life nouns that we may call the "fencenouns". She was motivated by considerations like this:

(4) a. Hans Müller's ranch has more fence than Alexis Sánchez's granja.

b. A fence can be cut in half and part of it moved, and then there are two fences.

As can be seen from (4-b), Rothstein's dual-life evidence is semantic in nature, as well as the syntactic (4-a), although even in (4-b) we see the syntactic point that 'fence' can be used with an indefinite determiner. Rothstein makes a similar case for other nouns, such as wire. And though she does not remark on the fact that she is testing nouns, as opposed to noun senses, one could plausibly argue that she in fact has kept the sense of 'fence' (and 'wire') constant in these examples, and that is borne out by our annotators, who were just looking at individual senses. Class 726 is the home of a number of such noun senses.

(5) a. cable\#2: a conductor for transmitting electrical or optical signals or electric power

b. cable\#3, cord\#1, ribbon\#4, rope\#1, thread\#1, wire\#1

Another identifiable subgroup of Class 726 is what we call the -sides dual life group. This group does not seem to have been identified in the earlier literature. Our annotators have determined that some sentence such as (6-a) is grammatical (and not due to counting the number of distinct seasides in the two locations), and as well, it is obvious that sentences like (6-b) are grammatical. And yet this is the same sense of seaside.

(6) a. California has more seaside than Oregon.

b. We spent our vacation on a seaside in southern England. 
Our data included only 4 such senses:

(7) a. seaside\#1: the shore of a sea or ocean regarded as a resort

b. hillside\#1, riverside\#1, roadside\#1

A final small group of the nominally-oriented dual-life senses we call the -land senses:

(8) a. marshland\#1: low-lying wet land with grassy vegetation; usually is a transition zone between land and water

b. forest\#2, marsh\#1, rainforest\#1, swamp\#1, wetland\#1

There are many other Nominally-Oriented duallife senses in Class 726 which do not manifest any of the preceding four types of meaning. The most common of the remaining ones are what we call "Kind-Instance" (or "Type-Token") in nature, usually where the kind-"meaning" is mass while the instance-"meaning" is count. There are 31 such senses, a few of which are:

(9) a. drought\#1: A shortage of rainfall."Farmers most affected by the drought hope that there may yet be sufficient rain early in the growing season"3

b. mockery\#3: humorous or satirical mimicry.

c. brunch\#1: combination breakfast and lunch; usually served in late morning

d. anticoagulant\#1: medicine that prevents or retards the clotting of blood

In (9-a) we have a general term, 'drought', which has many instances such as exemplified in A drought has bedevilled California since 1999. So this seems a straightforward example of our Nominally-Oriented Kind-Instance duallife senses. Things are maybe a little less obvious with (9-b), but it seems plausible to claim that it is designating a kind or sort of linguistic activity, and each particular case of a humorous or satirical mimicry is a mockery. Here we are not saying that the particular cases are the result of the activity, but rather that they exemplify the kind, mockery. With (9-c) one might wonder why it was not classified as an example of the Food subtype. In our opinion this is because brunch is not the "con-

\footnotetext{
${ }^{3}$ The only other sense of drought in WordNet concerns any prolonged shortage: "When England defeated Pakistan it ended a ten-year drought".
}

tainer" that has the mass-stuff as its makeup, in the way that a chicken is a container for chicken(meat). Instead, brunch is the name for a kind of activity, and each one of its manifestations is a brunch.

As we noted above, most senses here make the general "meaning" be mass, and the more individual-denoting "meaning" be count. But there are exceptions to this. Anticoagulant\#1, for example, seems to be a mass sense for the stuff that is put into one's body to retard blood clotting, that is, this "meaning" picks out the physical manifestation of the kind term (akin to the individualdenoting "meaning" of the more usual terms). But this makes this manifestation "meaning" be mass, and the count sense seems to be a "sorting" meaning, that is, the kind "meaning" is count. Thus, "an anticoagulant' names a kind or sort of stuff, rather like 'a beer' can name a type/kind/sort of beer. So 'anticoagulant' without an indefinite article names (something like) the stuff that is, in some particular case, doing the work of anti-coagulating.

\subsection{Verbally-Oriented Noun Senses}

There is a long-standing tradition claiming that "event nouns" ${ }^{4}$ are ambiguous between a "meaning" that describes an activity, action, event, or process, on the one hand, and a "meaning" that describes the result of that activity, action, event, or process, on the other hand. Some clear examples of this in the literature are:

(10) a. collection: the activity of gathering together a group of items vs. the group that is thus gathered.

b. invention: the process of generating some new type of thing vs. the actual kind of thing that has been generated.

Although some linguists would say that the fact that one "meaning" is predictable from the other "meaning", and so they shouldn't both be entered in the lexicon (e.g., (Payne and Huddleston, 2002, p. 337)), sometimes these two "meanings" are distinguished as separate senses in WordNet (and in dictionaries more generally), but sometimes not. For example, in WordNet we find

\footnotetext{
${ }^{4}$ We use 'event' to name this group, even though there are nouns that don't seem to be derived from event verbs nor do they intuitively designate the occurrence of some event. For example, some are actions, activities, achievements, processes, etc..
} 
(11) a. collection\#1: several things grouped together or considered as a whole

b. collection\#2: a publication containing a variety of works

c. collection\#3: a request for a sum of money

d. collection\#4: the act of gathering something together

Here we see the two "meanings" separated as different senses (senses \#1 vs. \#4). On the other hand, sometimes the two "meanings" are merged into the same sense. WordNet gives

(12) a. burglary\#1: entering a building unlawfully with intent to commit a felony or to steal valuable property ${ }^{5}$

b. emission\#1: the act of emitting; causing to flow forth ${ }^{6}$

c. amplification\#1: addition of extra material or illustration or clarifying detail ${ }^{7}$

Here we see that burglary\#1 describes both the activity of burgling and also the result of the activity (a burglary). emission\#1 describes both the event of causing something to flow, and also the result of that event (an emission). Similarly, amplification\# 1 describes both the activity of adding extra material and the result of doing so. The former "meanings" are mass(-like) while the latter are count(-like), as the examples in (13)-(15) suggest.

(13) a. Burglary is not a difficult activity to carry out.

b. The Müllers' house suffered a burglary last night.

(14) a. Methane emission in coal mines is a serious health issue.

b. The cause of the miners' deaths was determined to be an emission of methane.

(15) a. The Opposition demanded amplification of the Prime Minister's remarks.

b. The Ministers for Foreign Affairs and of

\footnotetext{
${ }^{5}$ This is the only sense identified in WordNet.

${ }^{6}$ Other senses of emission focus on the kind of material that is released by an emission; one sense even picks out a specific subtype of that sort of emission, namely that of water from a pipe.

${ }^{7}$ The other two senses of 'amplification' indicate rather different features: amplification\#2: 'the amount of increase in signal power or voltage or current expressed as the ratio of output to input'; amplification\#3: '(electronics) the act of increasing voltage or power or current'.
}

Defense each provided an amplification of the Prime Minister's remarks.

There is another relation that is, in a way, "between" these the categories of NominallyOriented Kind-Instance and Verbally-Oriented Event-Result. It happens when an event-noun (or state- or process-noun) designates a general term for a kind or type that has instances or tokens called by the same name. Note that these are instances or tokens of the kind, and not the result of the event. In such cases, the event/state/process "meaning" is usually or naturally seen as mass, while the instance "meaning" seems usually or naturally to be count. For example,

(16) a. fantasy\#1: imagination unrestricted by reality; "a schoolgirl fantasy"

b. litigation\#1: a legal proceeding in a court; a judicial contest to determine and enforce legal rights

c. silence\#1: the state of being silent (as when no one is speaking); "there was a shocked silence"; "he gestured for silence"

These cases seem to suggest that we have a general term ('fantasy', 'litigation', 'silence') denoting a type or kind, which in turn has many instances. The instances are thus not effects of these kinds. For example, "a schoolgirl fantasy", "a legal proceeding", "a shocked silence" are all instances of their respective kinds, but not effects of them. Nonetheless, it seems clear that these all display the fact that the ultimate source of these nounsenses is a verb: fantasize, litigate, silence, whose WordNet senses are: "to portray in the mind", "to engage in legal proceedings", "to cause to be quiet or not talk". So such senses seem best classified as Verbally-Oriented Kind-Instance senses, and as we said, should be seen as forming a sort of middle ground between the Nominally-Oriented KindInstance senses and the Verbally-Oriented EventAct senses. And so we call these senses VerballyOriented Kind-Instance dual-life.

But in many cases it is difficult to determine whether we have a case of the Verbally-Oriented Act-Result relation or of the Verbally-Oriented Kind-Instance relationship. For instance, with each of the senses identified in (17), it seems that there is no good reason to choose between viewing the relationship as an Event-Result or as a Kind- 
Instance:

(17) a. eccentricity\#1: strange and unconventional behavior

b. idealization\#2: (psychiatry) a defence mechanism that splits something you are ambivalent about into two representations - one good and one bad

c. imperfection\#1: the state or an instance of being imperfect

Is eccentricity\# 1 , for instance, the name for a process, activity, or force, etc., that brings about an eccentricity as a result? Or is it instead the name of a kind or type of force (etc.) which has instances that are called eccentricities? What about idealization\#2? Or imperfection\#1? In the latter case it is explicitly defined as "either a state or an instance" and so even the definition explicitly leaves room for either interpretation. There seems to be no good reason to view any of these (and others) in one way or the other. About all that can be said is that these are Verbally-Oriented, but we can't further determine which subtype they manifest. Or maybe better put: they in fact do manifest both types equally.

Examples such as these make one want to go back to the earlier examples of Event-Result and Kind-Instance and reanalyze them also, making it become easier to see them too as perhaps exemplifying both ways in which a single sense can be simultaneously mass and count. In fact, we are tempted to say that there is some sort of "continuum" or continuity between the two ways - in the same way that one can order wavelengths of light so as to display a continuum between blue and green.

The largest subgroup in the 726 Dual-Life Class is the Verbally-Oriented Event-Result senses. (Although keep in mind that many of these also manifest at least a degree of Verbally-Oriented KindInstance "meaning".) There are 70 such senses in this Class; 55 of them are senses of -tion nominalizations from activity verbs, 2 are -ment nominalization, 3 are -ing nominalizations, and there are 15 others. A few examples of each of these types senses are in $(18-a)-(18-d)$.

(18) a. acclimation\#1: adaptation to a new climate (a new temperature or altitude or environment); deception\#1: the act of deceiving; insertion\#2: the act of putting one thing inside another; elimination\#4: the act of removing an unknown mathematical quantity by combining equations;

b. embellishment\#1: elaboration of an interpretation by the use of decorative (sometimes fictitious) detail; infringement\#1: an act that disregards an agreement or a right.

c. borrowing\#1: the appropriation (of ideas or words etc) from another source; ending\#2: the act of ending something.

d. analysis\#2: the abstract separation of a whole into its constituent parts in order to study the parts and their relations; burglary\#1: entering a building unlawfully with intent to commit a felony or to steal valuable property; dispersal\#1: the act of dispersing or diffusing something; influx\#1; revival\#1; war\#1

A group that is somewhat smaller than the justmentioned Verbally-Oriented Event-Result senses is that of Verbally-Oriented Kind-Instance senses, with 18 members. Unlike the Verbally-Oriented Event-Result senses where one "meaning" is a name for the kind of activity and the other "meaning" is a name for a result of that activity, here we have a "meaning" as a name for the kind of activity and the other "meaning" is a name for tokens or instances of that activity, rather than a result of that activity. But unlike the Nominally-Oriented Kind-Instance noun-senses, these Verbally-Oriented senses clearly rely on a sense of a verb and not derived from a noun-sense. 10 of these noun-senses are -tion nominalizations, one formation is from each of -ing, -ship, -ment, while five are otherwise derived. A handful of the tion-formations are in (19-a), the -ing, -ship, -ment formations are in (19-b), while the others are in (19-c).

(19) a. elaboration\#3: a discussion that provides additional information; intonation\#1: rise and fall of the voice pitch; recrimination\#1: mutual accusations;

b. looting\#1: plundering during riots or in wartime; displacement\#4: (chemistry) a reaction in which an elementary substance displaces and sets free a constituent element from a compound; friendship\#1: the state of being friends 
c. curvature\#1: (medicine) a curving or bending; often abnormal; fantasy\#1: imagination unrestricted by reality; genocide\#1: systematic killing of a racial or cultural group; silence\#1: the state of being silent (as when no one is speaking); tribute\#1: something given or done as an expression of esteem

Here that there is always a verb-oriented situation, where the activity it describes gives rise to a mass general name for that activity, and the results are described by a count "meaning" of the same name.

\subsection{Borderline Cases}

Our rationales for the distinction between KindInstance noun-senses and Event-Result nounsenses (when they are both Verbally-Oriented) is this:

If the event's happening suggests a cause for the result, then it is a case of Event-Result "meaning".

When the event seems not to play any role in the formation, causation, occurrence, or existence of the object in question, then it is a Kind-Instance "meaning".

We think that these two explanations can merge into one another. E.g., in (22) it seems that the event is causing an instance to occur, and so we classify it in the Event-Result group, even though one can also see that the so-called result maybe is just an instance of the kind indicated by the event.

(22) a. insertion\#2: the act of putting one thing into another

b. encryption\#1: the activity of converting data or information into code

c. re-creation\#1: the act of creating again.

d. amelioration\#1: the act of relieving ills and changing for the better

Although the examples in (22) are most naturally seen as cases where an event causes some result, they could also be seen the other way. So, it seems natural to say that putting one thing into another causes there to be some result - an insertion. The activity of converting data to code (encryption) brings about an encryption of the data. But on the other hand, one might say that the abstract kind (or type), insertion, has various specific physical manifestations - various instances or tokens of that type. As we said, we think the former is more natural here and in the other members of (22), but it also seems that the latter understanding is certainly possible.

But when there was no salient particular causation involved, and it was merely a matter of some abstract kind (or concept) which is then said to be instantiated in a particular situation, we labelled it as Verbally-Oriented Kind-Instance, as in (23):

(23) a. elaboration\#3: a discussion that provides additional information

b. fantasy\#1: imagination unrestricted by reality; "a schoolgirl fantasy"

c. retraction\#1: a disavowal or taking back of a previous assertion

d. genocide\#1: systematic killing of a racial or cultural group

Here it seems more natural to think that the noun is describing some (abstract) kind or type, and the count interpretation is a manifestation of that type. In this way, 'elaboration' seems to us to describe a type of speech act, and its manifestations or instantiations will be this or that elaboration. (Of course, one might also say that an act of elaboration is an action which will result in some specific elaboration, which makes the Event-Result reading become more prominent.)

However, there seem to be various noun senses in Class 726 that are Verbally-Oriented, but for which we find it impossible to decide whether they are more clearly Event-Result or more clearly Kind-Instance. Probably the best thing to say about them is that they are both Verbally-Oriented Event-Result and VerballyOriented Kind-Instance to the same extent. Here are two representatives:

(24) a. defection\#1: withdrawing support or help despite allegiance or responsibility

b. eccentricity\#1: strange and unconventional behavior

Finally, we see a very few noun-senses that seem to be equally Verbally- and Nominally-Oriented:

(25) a. curve\#1: the trace of a point whose direction of motion changes

b. poop\#1: obscene terms for feces 
c. regret\#1: sadness associated with some wrong done or some disappointment

\section{Concluding Remarks}

We have offered some theoretical considerations for favouring an analysis of (dictionary-defined) senses of nouns, rather than the nouns themselves, as the locus for explaining why a NP is +MAss or +COUnT. We have also offered empirical evidence in the form of a large repository of carefully annotated noun-senses. These annotated senses can be analyzed to determine which individual ones of them can be used only in +COUNT NPs, or only in +MASS NPs, or in both + COUNT and + MASS NPs, or are not usable in either + MAss or +COUNT NPs. This paper in particular discussed a class of senses of the third of these varieties: "Dual-life" senses those individual meanings that can be used in both +MASS and +COUNT NPs.

We view the current undertaking as a necessary step in providing a complete semantic analysis of +MASs and +COUnT NPs. Such an account requires both the underlying meanings of the component nouns, and also the semantic effect of the syntactic method of forming the NP (that is, the giving the meaning of the NP) from the noun's meaning. However, without a detailed account of the wide range of senses of the component nouns, it will be impossible to give the desired group of semantic rules. And without that, there would be no hope for a compositional account of these phenomena.

We encourage other researchers to investigate the resources available with the Bochum English Countability Lexicon (BECL). The BECL 2.1 database is publicly available at http:// count-and-mass.org.

\section{Acknowledgments}

We gratefully acknowledge the Alexander von Humboldt Foundation for an Anneliese-Maier prize and grant to Pelletier, and the Deutsche Forschungsgemeinschaft (KI-759/5) grant to Kiss, for their support of the work reported here and our other reports.

\section{References}

Allan, K. (1980). Nouns and countability. Language 56, 541-567.

Artstein, R. and M. Poesio (2008). Inter-coder agreement for computational linguistics. Computational Linguistics 34, 555-596. http://aclweb. org/anthology/J08-4004.

Baldwin, T. and F. Bond (2003). Learning the countability of English nouns from corpus data. In Proc. of the 41st Annual Meeting of the Association for Computational Linguistics. http://aclweb. org/anthology/PS03-1059.

Barner, D., S. Inagaki, and P. Li (2009). Language, thought, and real nouns. Cognition 11, 329-344.

Bond, F. (2005). Translating the Untranslatable: A Solution to the Problem of Generating English Determiners. Stanford: CSLI Press.

Bunt, H. (1985). Mass Terms and Model Theoretic Semantics. Cambridge: Cambridge UP.

Chen, D. and C. Manning (2014). A fast and accurate dependency parser using neural networks. In Proceedings of EMNLP 2014. doi: 10.3115/v1/D141082.

Grimm, S. (2014). Individuating the Abstract. In U. Etxeberria, A. Fălăuş, A. Irurtzun, and B. Leferman (Eds.), Proceedings of Sinn und Bedeutung 18, Bayonne and Vitoria-Gasteiz, pp. 182-200.

Ide, N. (2008). The Amercian National Corpus: Then, now, and tomorrow. In M. Haugh, K. Burridge, J. Mulder, and P. Peters (Eds.), Selected Proceedings of the 2008 HCSNet Workshop on Designing the Australian National Corpus: Mustering Languages, Summerville, MA. Cascadilla Proceedings Project.

Ide, N. and K. Suderman (2004). The Amercian National Corpus first release. In Proceedings of the Fourth Language Resources and Evaluation Conference (LREC), Lisbon, pp. 1681-1684. http: / / aclweb.org/anthology/L04-1313.

Katz, G. and R. Zamparelli (2012). Quantifying count/mass elasticity. In J. Choi (Ed.), Proceedings of the 29th West Coast Conference on Formal Linguistics, Somerville, MA, pp. 371-379. Cascadilla Proceedings Project.

Kiss, T., F. J. Pelletier, H. Husić, and J. Poppek (2016). A sense-based lexicon of count and mass expressions: The Bochum English countability lexicon. In Proceedings of LREC 2016, Portoroz, Slovenia. http: / / aclweb.org/anthology/L16.

Kiss, T., F. J. Pelletier, and T. Stadtfeld (2014). Building a reference lexicon for countability in English. In Proceedings of the Ninth LREC 2014, Reykjavik. http://aclweb.org/anthology/ L14-1312. 
Krifka, M. (1991). Massennomina. In A. von Stechow and D. Wunderlich (Eds.), Semantics: An International Handbook of Contemporary Research, pp. 399-417. Berlin: Mouton de Gruyter.

Kulkarni, R., S. Rothstein, and A. Treves (2013). A statistical investigation into the cross-linguistic distribution of mass and count nouns: Morphosyntactic and semantic perspectives. Biolinguistics 7, 132168 .

McCawley, J. (1975). Lexicography and the countmass distinction. In Berkeley Linguistic Society, Vol. 1, pp. 314-321. Reprinted in J. McCawley (ed.) Adverbs, Vowels, and Other Objects of Wonder, Univ. Chicago Press, Chicago, 1979, pages 165-173.

Middleton, E., E. Wisniewski, K. Trindel, and M. Imai (2004). Separating the chaff from the oats: Evidence for a conceptual distinction between count noun and mass noun aggregates. Journal of Memory and Language 50, 371-394.

Miller, G. (1995). WordNet: A lexical database for English. Communications of the ACM 38, 39-41.

Miller, G. and C. Fellbaum (2007). WordNet then and now. Language Resources and Evaluation 41, 209 214. doi: 10.1007/s10579-007-9044-6.

Payne, J. and R. Huddleston (2002). Nouns and noun phrases. In R. Huddleston and G. K. Pullum (Eds.), The Cambridge Grammar of the English Language, pp. 323-523. Cambridge, UK: Cambridge UP.

Pelletier, F. J. (1975). Non-singular reference: Some preliminaries. Philosophia 5, 451-465. Reprinted in (Pelletier, 1979, pp. 1-14).

Pelletier, F. J. (Ed.) (1979). Mass Terms: Some Philosophical Problems. Dordrecht: Kluwer Academic Pub.

Pustejovsky, J. (1991). The generative lexicon. Computational Linguistics 17, 409-441. http:// aclweb.org/anthology/J91-4003.

Quine, W. (1960). Word and Object. Cambridge, MA: MIT Press.

Rothstein, S. (2010). Counting and the mass-count distinction. Journal of Semantics 27, 343-397.

Schmidtke, D. and V. Kuperman (2016). Mass counts in world Englishes: A corpus linguistic study of noun countability in non-native varieties of english. Corpus Linguistics and Linguistic Theory 12. doi: 10.1515/clit-2015-0047. 